\title{
Assessment the relationship between TSH and selected anthropometric parameters - preliminary report
}

\author{
Authors ${ }^{1}$ Aneta Koszowska, ${ }^{2}$ Justyna Nowak, ${ }^{3}$ Anna Brończyk- Puzoń, ${ }^{4}$ Paweł Jagielski, ${ }^{5}$ Anna \\ Dittfeld, ${ }^{6}$ Karolina Kulik, ${ }^{7,8}$ Barbara Zubelewicz-Szkodzińska
}

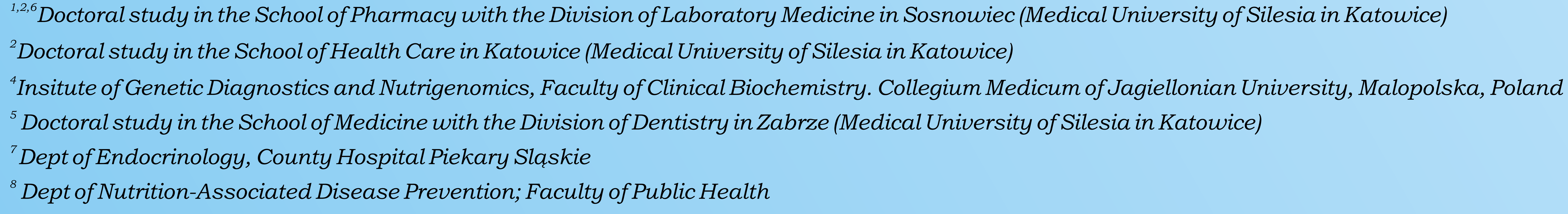

${ }^{8}$ Dept of Nutrition-Associated Disease Prevention; Faculty of Public Health

\section{ECE2014 3-7 May 2014 Wrocław, Poland}

\section{Introduction}

The majority of secreted hormones influence the whole body, its weigh and constitution as the results of ongoing metabolism. Scientific studies have reported the existence of relationship between the TSH and selected anthropometric parameters such as BMI or body weight.

\section{Aim}

The aim of the study was to assess the relationship between the value of TSH and selected anthropometric parameters in the group of endocrine patients.

Table 1. Characteristic of the whole analyzed groupdescriptive statistics

\section{Materials and methods}

The study involved 87 patients with thyroid disorders who were admitted to the Endocrinology Department in 2013. We excluded patients treated with statins and patients with TSH values below $0,35 \mathrm{uIU} / \mathrm{ml}$. Blood samples for TSH concentration were collected from patients in the morning. Anthropometric parameters were measurement in the morning in accordance with generally accepted methodology. The collected data were statistically analyzed with Spearman Rank Correlation . $\mathrm{a}=0,05$.

\section{Results}

Characteristic of the group Table 1 . In the preliminary stage of the study we did not find any statistically significant correlation in TSH concentration and waist circumference $(R=0,16 ; p=0,1221)$, hip circumference $(R=-$ $0,02 ; p=0,8023)$, BMI score $(R=0,04 ; p=0,6873)$, percentage of body fat $(\mathrm{R}=0,01 ; \mathrm{p}=0,8807)$, lean body mass $(\mathrm{R}=-0,01 ; \mathrm{p}=0,8915)$ and muscle mass $(R=-0,02 ; r=0,8296)$. There were observed positive significant correlation between the TSH concentration and WHR index $(R=0,25 ; p=0,0160)$, and between TSH and WHtR ratio $(R=0,21 ; p=0,0420)$. In addition the results showed a negative correlation between TSH concentration and the strength of hand $(R=-0,24 ; p=0,0215)$. Table 2 .

\section{Conslusions}

The thyroid function (based on screening TSH exam) could affect body constitution and muscle strength. Enlargement of the studied group is necessary to confirm the observation.
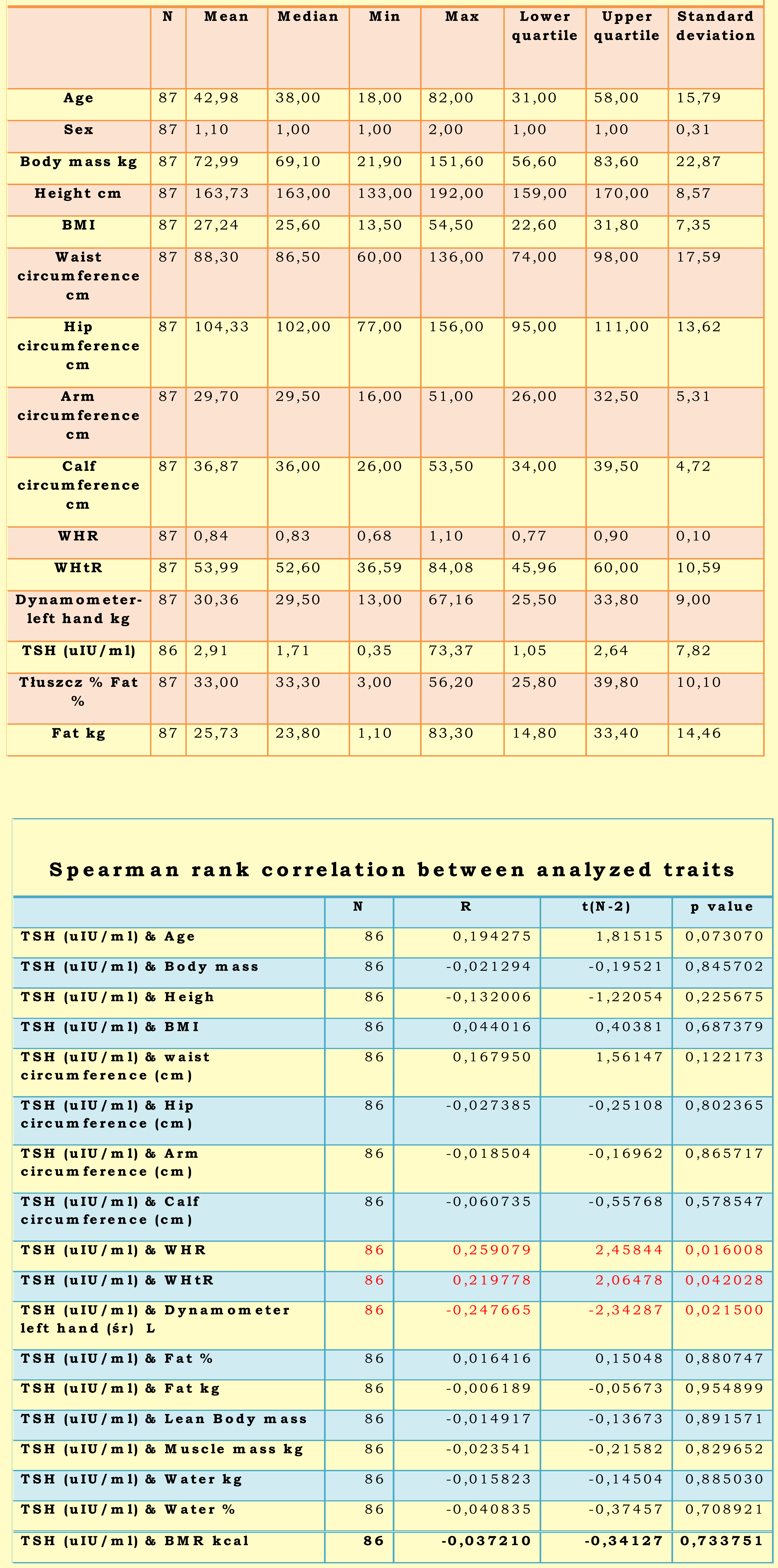